\title{
Synthesis, Antioxidant and Antimicrobial Activities of a Novel Series of Chalcones, Pyrazolic Chalcones, and Allylic Chalcones
}

\author{
Tan Nhut Doan, Dao Thanh Tran
}

Department of Medicinal Chemistry, School of Pharmacy, University of Medicine and Pharmacy, Ho Chi Minh City, Vietnam. Email: tan.doan@uqconnect.edu.au

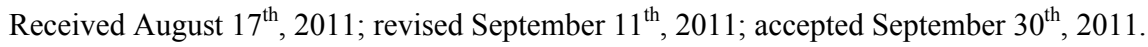

\begin{abstract}
A new series of chalcones (4a-c) and allylicchalcones (11a-b) have been prepared by the Claisen-Schmidt condensation. A novel series of pyrazolicchalcones (5a-c) have been synthesized by the reaction of respective chalcones $(4 a-c)$ and hydrazine hydrate. The structures of the compounds were confirmed by spectral data (infrared spectroscopy and ${ }^{1} \mathrm{H}$ nuclear magnetic resonance). All of the compounds (4/5a-c and 11a-b) have been tested for their antimicrobial activities (agar disc-diffusion method) and antioxidant activities (1,1-biphenyl-2-picrylhydrazyl free radical scavenging method). The test compounds failed to show antibacterial properties $(4 a-c, 5 b$, and $11 a-b)$ or exhibited such properties poorly $(5 a$ and $5 c)$. None of the test compounds displayed antifungal properties. Of the compounds tested, compounds $5 a-c$ and $11 a-b$ exhibited promising antioxidant activities.
\end{abstract}

Keywords: AllylicChalcones, Antioxidant Activity, Antimicrobial Activity, PyrazolicChalcones

\section{Introduction}

Flavonoids comprise a large family of plant-derived polyphenolic compounds classified as anthocyanidins, flavonols, chalcones, aurones, flavanones, isoflavones, flavans, flavanonols, flavanols, and flavones differencing from each other in their structural group arrangements [1]. Chalcone, an important intermediate of flavonoid synthetic pathway, has been shown to exhibit diverse biological and pharmacological activities such as anticancer, antioxidant, anti-inflammatory, antimicrobial, antiallergic, and antimalarial properties [2-4].

Oxidative damage which is implicated in various pathological events such as cancer and aging is induced by free radicals and reactive oxygen species [5]. Antioxidants are the compounds that prevent such oxidative damage due to their free radical scavenging ability [5]. In chalcones, such ability is attributable to phenolic-OH group attached to the ring structure [6]. Chalcones with antioxidant activity (and compounds with such activity in general) have been demonstrated to have anticancer, anticardiovascular, anti-inflammatory, and many other activeties $[7,8]$. As such, they have gained immense interest from bioorganic and medicinal chemistry research.
Synthesized chalcones holding allylic substitutions and pyrazolicchalcones were recently reported as potent antimicrobial and antioxidant agents [9-12]. In addition, the presence of enone function in chalcones having pyrazole moiety has been found to enhance the biological activity [13].

Prompted by all these observations, we report herein the synthesis, antioxidant and antimicrobial activities of novel chalcones, pyrazolicchalcones and allylicchalcones.

\section{Chemistry}

In order to obtain pyrazolicchalcones 5a-c, the corresponding chalcones $4 \mathrm{a}-\mathrm{c}$ were prepared by the ClaisenSchmidt condensation of respective acetophenones (1) and benzaldehydes (2) by the known literature method [14]. Subsequently, the corresponding pyrazolicchalcones 5a-c were prepared by the addition of the obtained chalcones 4a-c to hydrazine hydrate in absolute ethanol (Scheme 1). All of the compounds were characterized by spectral data (infrared spectroscopy [IR] and ${ }^{1} \mathrm{H}$ nuclear magnetic resonance [NMR]).

The synthetic procedures and reaction conditions for allylicchalcones 11a-b are illustrated in Scheme 2. 2-Allyloxybenzaldehyde (8) was prepared by the reaction of 


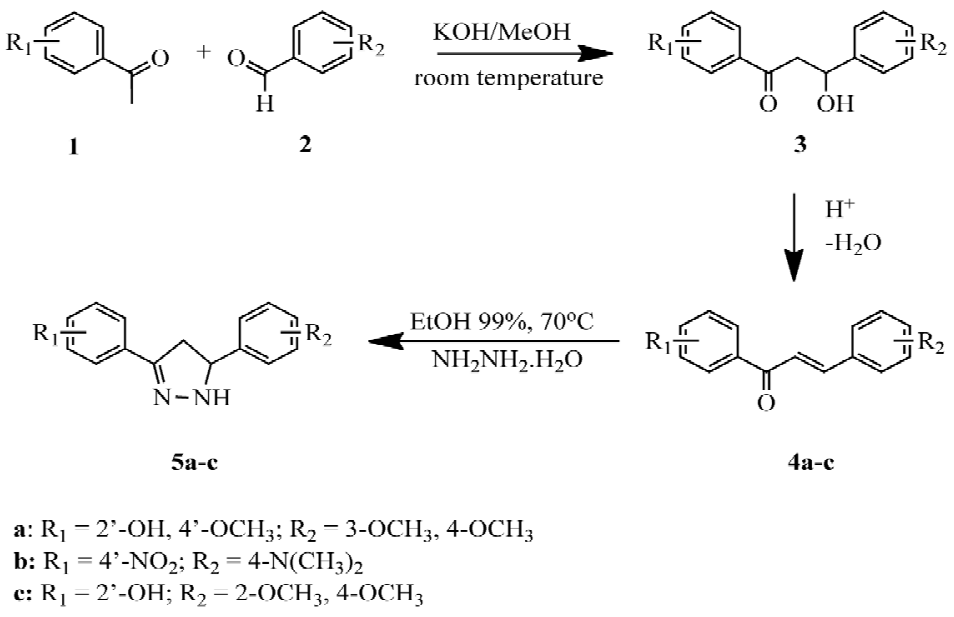

Scheme 1. Synthesis of the novel chalcones $4 a-c$ and pyrazolic chalcones 5 a-c.

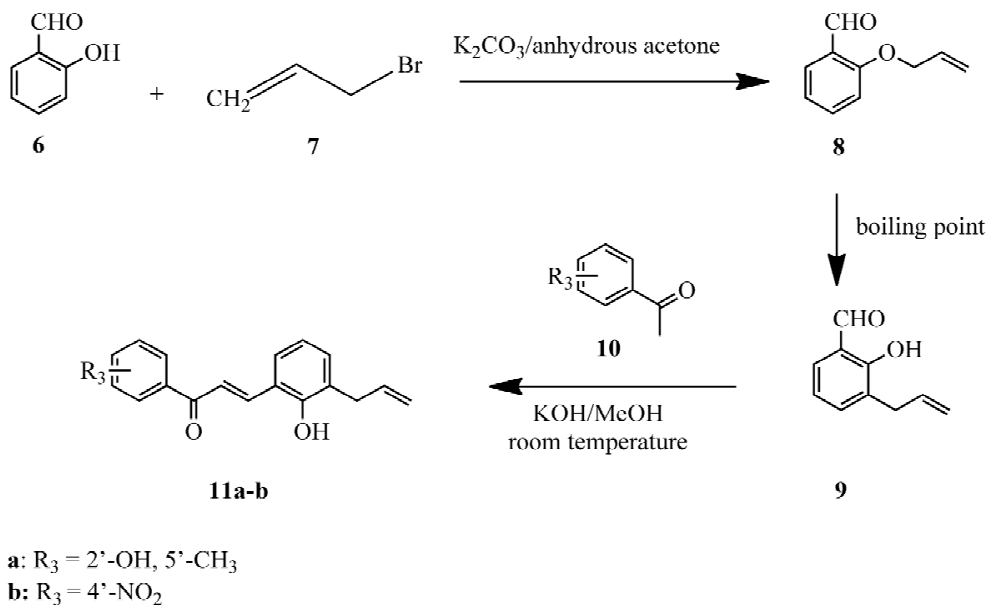

Scheme 2. Synthesis of the novel allylic chalcones 11a-b.

salicylaldehyde (6) and allyl bromide (7) in the presence of potassium carbonate in anhydrous acetone. Subsequently, 2-hydroxy-3-allylbenzaldehyde (9) was obtained by the Claisen thermal rearrangement [15]. The corresponding allylicchalcones $11 \mathrm{a}-\mathrm{b}$ were then prepared by the Claisen-Schmidt condensation of the obtained 2-hydroxy-3-allylbenzaldehyde (9) and respective acetophenones (10). The spectral data (IR and ${ }^{1} \mathrm{H}$ NMR) were used to ascertain the structures of the compounds.

\section{Biology}

\subsection{Antimicrobial Activity}

Compounds 4/5a-c and 11a-b were tested for their in vitro antimicrobial properties against the Gram-positive bacteria Methicillin-resistant Staphylococcus aureus (MRSA) (ATCC 43300), Methicillin-sensitive Staphylococcus aureus (MSSA) (ATCC 25923), Streptococcus faecalis (ATCC 10541), the Gram-negative bacteria Es- cherichia coli (ATCC 25922), Pseudomonas aeruginosa (ATCC 27853), fungi Candida albicans (ATCC 10201) and Candida albicans 955 using conventional agar discdiffusion method [16]. The minimum inhibitor concentrations (MICs) were determined using micro-dilution susceptibility method [17]. Sulfamethoxazole and Ketoconazole were the reference drugs for antibacterial and antifungal testing respectively. The observed data on the antimicrobial testing are presented in Tables 1-2.

\subsection{Antioxidant Activity}

Compounds 4/5a-c and 11a-b were assessed for antioxidant activity using 1,1-biphenyl-2-picrylhydrazyl (DPPH) radical scavenging method [18]. The observed data on the antioxidant activity are given in Table 3 .

\section{Results and Discussion}

We have synthesized a series of chalcones 4a-c, pyra- 
zolicchalcones 5a-c and allylicchalcones $11 \mathrm{a}-\mathrm{b}$ with appropriate nucleophiles using the procedures presented in Schemes 1 and 2.

\subsection{Antimicrobial Activity}

The results of antimicrobial testing of compounds $4 / 5 \mathrm{a}-\mathrm{c}$ and 11a-b are presented in Tables 1-2. The results indicated that compounds $4 \mathrm{a}-\mathrm{c}, 5 \mathrm{~b}$ and $11 \mathrm{a}-\mathrm{b}$ did not exhibit antibacterial properties whereas compounds $5 \mathrm{a}$ and $5 \mathrm{c}$ displayed poor antibacterial activity towards Gram-positive bacteria showing inhibitor zones between 10 and 16 $\mathrm{mm}$ and MIC values higher than $256 \mu \mathrm{g} / \mathrm{ml}$ for Streptococcus faecalis and $512 \mu \mathrm{g} / \mathrm{ml}$ for MSSA and MRSA compared to Sulfamethoxazole. These compounds (5a and $5 \mathrm{c}$ ), however, did not exhibit antibacterial activity against Gram-negative bacteria. None of the synthesized compounds (4/5a-c and 11a-b) showed antifungal properties.

\subsection{Antioxidant Activity}

The antioxidant activity of compounds 4/5a-c and 11a-b were tested using DPPH radical scavenging method [18]. Except for compounds 4a-c which failed to show such activity, all of the other test compounds (5a-c and 11a-b) exhibited good antioxidant properties, with the strongest being observed in compound 5a (Table 3). However, all the synthesized compounds were less potent than vitamin $\mathrm{C}$ as the reference. The potencies for the antioxidant activity of the test compounds to the reference drug are in the following order: Vitamin $\mathrm{C}>5 \mathrm{a}-\mathrm{b}>5 \mathrm{c}>11 \mathrm{a}-\mathrm{b}$.

\section{Conclusions}

In summary, we have synthesized a series of novel chalcones, pyrazolicchalcones and allylicchalcones. We have also reported antimicrobial and antioxidant evaluations of these compounds. All the synthesized compounds showed poor antimicrobial properties or did not show such properties. A good antioxidant activity was observed in pyrazolicchalcones and allylicchalcones.

\section{Experimental}

\subsection{Chemistry}

All chemicals were purchased from commercial suppliers, and used without further purification. All solvents used for reaction were freshly distilled from proper dehydrateing agents. Melting points were determined in open capillaries on a Gallenkamp Melting Point Apparatus and are uncorrected. The purity of the compounds was checked by thin layer chromatography (TLC) (silica gel H, n-hexaneacetone $3: 1)$. The IR spectra were performed on a Shimadzu FTIR 8101 spectrometer in potassium bromide

Table 1. Antimicrobial activity of 4/5a-c and 11a-b.

\begin{tabular}{|c|c|c|c|c|c|c|c|}
\hline \multirow{3}{*}{ Compound } & \multicolumn{7}{|c|}{ Zone of inhibition at concentration of $1024 \mu \mathrm{g} / \mathrm{ml}(\mathrm{mm})$} \\
\hline & \multicolumn{3}{|c|}{ Gram-positive bacteria } & \multicolumn{2}{|c|}{ Gram-negative bacteria } & \multicolumn{2}{|c|}{ Fungi } \\
\hline & MSSA & MRSA & $\mathrm{SF}$ & $\mathrm{EC}$ & PA & CA 10201 & CA 955 \\
\hline $4 \mathbf{a}$ & - & - & - & - & - & - & - \\
\hline $4 \mathbf{b}$ & - & - & - & - & - & - & - \\
\hline $4 c$ & - & - & - & - & - & - & - \\
\hline $5 \mathbf{a}$ & 12 & 14 & 11 & - & - & - & - \\
\hline $5 \mathbf{b}$ & - & - & - & - & - & - & - \\
\hline $5 c$ & 10 & 16 & 14 & - & - & - & - \\
\hline $11 \mathrm{a}$ & - & - & - & - & - & - & - \\
\hline $11 \mathrm{~b}$ & - & - & - & - & - & - & - \\
\hline Sulfamethoxazole & 34 & 36 & 38 & 35 & 33 & - & - \\
\hline Ketoconazole & - & - & - & - & - & 41 & 39 \\
\hline Control (DMSO) & - & - & - & - & - & - & - \\
\hline
\end{tabular}

Data represent the mean of three replicates. MSSA-Methicillin-sensitive Staphylococcus aureus(ATCC 25923); MRSAMethicillin-resistant Staphylococcus aureus(ATCC 43300); SF—Streptococcus faecalis (ATCC 10541); EC—Escherichia coli (ATCC 25922); PA-Pseudomonas aeruginosa(ATCC 27853); CA 10201—Candida albicans (ATCC 10201); CA 955-Candida albicans 955; DMSO — dimethyl sulfoxide. (-) indicates "not detected". 
Table 2. Minimum inhibitor concentration (MIC), $\mu \mathrm{g} / \mathrm{ml}$ of $5 \mathrm{a}$ and $5 \mathrm{c}$.

\begin{tabular}{cccc}
\hline \multirow{2}{*}{ Compound } & \multicolumn{3}{c}{ Minimum inhibitor concentration (MIC) $(\mu \mathrm{g} / \mathrm{ml})$} \\
\cline { 2 - 4 } & \multicolumn{3}{c}{ Gram-positive bacteria } \\
\cline { 2 - 4 } & MSSA & MRSA & SF \\
\hline $5 \mathbf{5 a}$ & 512 & 512 & 256 \\
Sulfamethoxazole & 4 & 512 & 256 \\
\hline
\end{tabular}

MSSA-Methicillin-sensitive Staphylococcus aureus (ATCC 25923); MRSAMethicillin-resistant Staphylococcus aureus (ATCC 43300); SF-Streptococcus faecalis (ATCC 10541).

Table 3. Antioxidant property of 4/5a-c and 11a-b.

\begin{tabular}{cc}
\hline Compound & $\%$ DPPH \\
\hline $\mathbf{4 a}$ & 0.00 \\
$\mathbf{4 b}$ & 0.00 \\
$\mathbf{4 c}$ & 0.00 \\
$\mathbf{5 a}$ & 89.64 \\
$\mathbf{5 b}$ & 89.27 \\
$\mathbf{5 c}$ & 77.14 \\
$\mathbf{1 1 a}$ & 41.00 \\
$\mathbf{1 1 b}$ & 40.90 \\
Vitamin $C^{*}$ & 97.92 \\
\hline
\end{tabular}

DPPH-1,1-biphenyl-2-picrylhydrazyl. *Standard substance.

$(\mathrm{KBr})$ pellets and the wave numbers were given in $\mathrm{cm}^{-1}$. The ${ }^{1} \mathrm{H}$ NMR spectra were run on a Bruker spectrometer operating at $500 \mathrm{MHz}$, using deuterated chloroform $\left(\mathrm{CDCl}_{3}\right)$ as solvent and tetramethylsilane (TMS) as internal standard. All chemical shifts are reported in parts per million (ppm) downfield relative to TMS on the $\delta$ scale. Data are reported as follows: chemical shift, multiplicity $(\mathrm{s}=$ single, $\mathrm{d}=$ double, $\mathrm{t}=$ triplet, $\mathrm{q}=$ quarter, $\mathrm{m}=$ multiplier, $\mathrm{br}=$ broad), coupling constant $(\mathrm{Hz})$ and integration.

\subsubsection{2-Hydroxy-4-methoxyacetophenone}

2,4-Dihydroxyacetophenone $(0.1 \mathrm{~mol})$, potassium carbonate $(0.3 \mathrm{~mol})$ and dimethyl sulphate $(0.09 \mathrm{~mol})$ in 20 $\mathrm{ml}$ anhydrous acetone were refluxed for 4 hours, monitoring by TLC with solvent system of n-hexane-acetone (3:1). After removing potassium carbonate, the reaction solution was evaporated in vacuo, giving solid. The solid was filtered, washed with water, dried, and crystalized from methanol to yield 2-hydroxy-4-methoxyacetophe0 none as gray solid with overall yield of $81 \%$. This prod- uct was used as the material for the synthesis of $4 \mathrm{a}$.

\subsubsection{2'-Hydroxy-3,4,4'-trimethoxychalcone (4a)}

2-Hydroxy-4-methoxyacetophenone (1.05 equivalence) and 3,4-dimethoxybenzaldehyde were dissolved in methanol. To the above mixture, potassium hydroxide ( 3 equivalence) was added in portions to give a blood-red solution. The reaction mixture was stirred at room temperature for 32 hours, during which 2'-hydroxy-3,4,4'-trimethoxychalcone (4a) precipitated as the potassium salt. The reaction mixture was poured into cold $1 \mathrm{~N}$ hydrochloride acid $(\mathrm{HCl})$ solution and was further added concentrated $\mathrm{HCl}(\mathrm{c}-\mathrm{HCl})$ until the solution became acidic. The resulting precipitate was filtered, washed with water, and crystalized from methanol to give product $4 \mathrm{a}$ as crystals. Yield $61 \%$. Light yellow solid, melting point $(\mathrm{mp})$ : $170^{\circ} \mathrm{C}, \mathrm{IR}(\mathrm{KBr}), v\left(\mathrm{~cm}^{-1}\right): 1633(\mathrm{C}=\mathrm{O}), 1564(\mathrm{C}=\mathrm{C}$ aryl $)$, 1126 (C-O). ${ }^{1} \mathrm{H}-\mathrm{NMR}\left(500 \mathrm{MHz}, \mathrm{CDCl}_{3}\right): \delta 13.50(\mathrm{~s}, 1$ $\mathrm{H}, \mathrm{Ar}-\mathrm{OH}), 7.85-7.82\left(\mathrm{~d}, J=15.5 \mathrm{~Hz}, 1 \mathrm{H}, \mathrm{H}_{\beta}\right), 7.84$ $7.82\left(\mathrm{~d}, J=8 \mathrm{~Hz}, 1 \mathrm{H}, \mathrm{H}_{6}\right), 7.44-7.41(\mathrm{~d}, J=15.5 \mathrm{~Hz}$, $\left.1 \mathrm{H}, \mathrm{H}_{\alpha}\right), 7.25-7.23\left(\mathrm{~d}, J=2 \mathrm{~Hz}, 8.5 \mathrm{~Hz}, 1 \mathrm{H}, \mathrm{H}_{6}\right), 7.16(\mathrm{~d}$, $\left.J=2 \mathrm{~Hz}, 1 \mathrm{H}, \mathrm{H}_{3}\right), 6.91-6.89\left(\mathrm{~d}, J=8 \mathrm{~Hz}, 1 \mathrm{H}, \mathrm{H}_{5}\right), 6.49$ - 6.47 (d, $J=2.5 \mathrm{~Hz}, 8.5 \mathrm{~Hz}, 1 \mathrm{H}, \mathrm{H}_{5}$ ), 6.47 (s, $J=2 \mathrm{~Hz}$, $\left.1 \mathrm{H}, \mathrm{H}_{2}\right), 3.96$ (s, $\left.3 \mathrm{H}, \mathrm{Ar}-\mathrm{OCH}_{3}\right), 3.93\left(\mathrm{~s}, 3 \mathrm{H}, \mathrm{Ar}-\mathrm{OCH}_{3}\right)$, $3.85\left(\mathrm{~s}, 3 \mathrm{H}, \mathrm{Ar}-\mathrm{OCH}_{3}\right)$.

\subsubsection{4'-Nitro-4-dimethylaminochalcone (4b)}

4-Nitroacetophenone (1.05 equivalence) and 4-dimethylaminobenzaldehyde were dissolved in methanol. To the above mixture, potassium hydroxide (3 equivalence) was added in portions to give a blood-red solution. The reaction mixture was stirred at room temperature for 32 hours, during which 4'-nitro-4-dimethylaminochalcone (4b) precipitated as the potassium salt. The reaction mixture was poured into cold $1 \mathrm{~N}-\mathrm{HCl}$ solution and was further added $\mathrm{c}-\mathrm{HCl}$ until the solution became acidic. The resulting precipitate was filtered, washed with water, and crystalized from methanol to give product $4 \mathrm{~b}$ as crystals. Yield $79 \%$. Light yellow solid, $\mathrm{mp}: 206^{\circ} \mathrm{C}$, IR $(\mathrm{KBr}), v\left(\mathrm{~cm}^{-1}\right)$ : $1647(\mathrm{C}=\mathrm{O}), 1518(\mathrm{C}=\mathrm{C}$ aryl $), 1258(\mathrm{C}-\mathrm{O}) .{ }^{1} \mathrm{H}-\mathrm{NMR}$ $\left(500 \mathrm{MHz}, \mathrm{CDCl}_{3}\right): \delta 7.93-7.90\left(\mathrm{~d}, J=15 \mathrm{~Hz}, 1 \mathrm{H}, \mathrm{H}_{\beta}\right)$, $7.93-7.91\left(\mathrm{~d}, J=1.5 \mathrm{~Hz}, 7.5 \mathrm{~Hz}, 1 \mathrm{H}, \mathrm{H}_{6}\right), 7.58-7.55(\mathrm{~d}$, $\left.J=8 \mathrm{~Hz}, 2 \mathrm{H}, \mathrm{H}_{2}, \mathrm{H}_{6}\right), 7.47-7.44\left(\mathrm{~d}, J=15 \mathrm{~Hz}, 1 \mathrm{H}, \mathrm{H}_{\alpha}\right)$, $7.47-7.44\left(\mathrm{t}, 1 \mathrm{H}, \mathrm{H}_{4}\right), 7.02-7.00\left(\mathrm{~d}, J=8 \mathrm{~Hz}, 1 \mathrm{H}, \mathrm{H}_{3}\right)$, $6.93-6.90\left(\mathrm{t}, 1 \mathrm{H}, \mathrm{H}_{4}\right), 6.71-6.69(\mathrm{~d}, J=2 \mathrm{~Hz}, 8 \mathrm{~Hz}, 2 \mathrm{H}$, $\left.\mathrm{H}_{3}, \mathrm{H}_{5}\right), 3.05\left(\mathrm{~s}, 6 \mathrm{H}, 2 \times \mathrm{CH}_{3}\right)$.

\subsubsection{2'-Hydroxy-2,4-dimethoxychalcone (4c)}

2-Hydroxyacetophenone (1.05 equivalence) and 2,4-dimethoxybenzaldehyde were dissolved in methanol. To the above mixture, potassium hydroxide ( 3 equivalence) was added in portions to give a blood-red solution. The reaction mixture was stirred at room temperature for 20 
hours, during which 2'-hydroxy-2,4-dimethoxychalcone (4c) precipitated as the potassium salt. The reaction mixture was poured into cold $1 \mathrm{~N}-\mathrm{HCl}$ solution and was further added c- $\mathrm{HCl}$ until the solution became acidic. The resulting precipitate was filtered, washed with water, and crystalized from methanol to give product $4 \mathrm{c}$ as crystals. Yield $59 \%$. Light yellow solid, mp: $111^{\circ} \mathrm{C}$, IR $(\mathrm{KBr}), v$ $\left(\mathrm{cm}^{-1}\right)$ : $1637(\mathrm{C}=\mathrm{O}), 1558(\mathrm{C}=\mathrm{C}$ aryl $), 1170(\mathrm{C}-\mathrm{O}) .{ }^{1} \mathrm{H}-$ NMR (500 MHz, $\left.\mathrm{CDCl}_{3}\right): \delta 13.10(\mathrm{~s}, 1 \mathrm{H}, \mathrm{Ar}-\mathrm{OH}), 8.14-$ $8.22\left(\mathrm{~d}, J=15.4 \mathrm{~Hz}, 1 \mathrm{H}, \mathrm{H}_{\beta}\right), 7.90-7.94(\mathrm{~d}, J=8.2 \mathrm{~Hz}$, $\left.1 \mathrm{H}, \mathrm{H}_{6}\right), 7.64-7.75\left(\mathrm{~d}, J=15.4,1 \mathrm{H}, \mathrm{H}_{\alpha}\right), 7.58-7.62(\mathrm{~d}$, $\left.J=8.6 \mathrm{~Hz}, 1 \mathrm{H}, \mathrm{H}_{6}\right), 7.44-7.52\left(\mathrm{~m}, J=8 \mathrm{~Hz}, 1 \mathrm{H}, \mathrm{H}_{4}\right)$, $6.99-7.03\left(\mathrm{~d}, J=8.6 \mathrm{~Hz}, 1 \mathrm{H}, \mathrm{H}_{5}\right), 6.93-6.97(\mathrm{~d}, J=8.4$ $\mathrm{Hz}, 1 \mathrm{H}, \mathrm{H}_{3}$ ) $, 6.55-6.59\left(\mathrm{~d}, J=7.6 \mathrm{~Hz}, 1 \mathrm{H}, \mathrm{H}_{5}\right), 6.5(\mathrm{ds}$, $\left.J=1.4 \mathrm{~Hz}, 1 \mathrm{H}, \mathrm{H}_{3}\right), 3.93\left(\mathrm{~s}, 3 \mathrm{H}, \mathrm{Ar}-\mathrm{OCH}_{3}\right), 3.87$ (s, 3H, $\left.\mathrm{Ar}-\mathrm{OCH}_{3}\right)$.

\subsubsection{2-(5-(3,4-Dimethoxyphenyl)-4,5-dihydro-1H- pyrazol-3-yl)-5-methoxyphenol (5a)}

2'-Hydroxy-3,4,4'-trimethoxychalcone (4a, $2 \mathrm{mmol}$ ) was dissolved in $100 \mathrm{ml}$ absolute ethanol. To this mixture, hydrazine hydrate $(4 \mathrm{mmol})$ was added dropwise at room temperature. The reaction mixture was refluxed for 7 hours, monitoring by TLC with solvent system of nhexane-acetone (3:1). The reaction mixture was cooled in ice bath. The resulting white solid was filtered and washed with cold water. The filtrate was extracted with ether and evaporated in vacuo yielding more solid. The solid was collected and crystalized from ethanol to yield 2-(5(3,4-dimethoxyphenyl)-4,5-dihydro-1H-pyrazol-3-yl)-5methoxyphenol (5a). White solid, $\mathrm{mp}: 115^{\circ} \mathrm{C}$, IR (KBr), $v\left(\mathrm{~cm}^{-1}\right): 3336(\mathrm{~N}-\mathrm{H}), 1591(\mathrm{C}=\mathrm{N}), 1155(\mathrm{C}-\mathrm{O}) .{ }^{1} \mathrm{H}-$ NMR $\left(500 \mathrm{MHz}, \mathrm{CDCl}_{3}\right): \delta 11.19$ (s, 1H, Ar-OH), 7.07 $7.06\left(\mathrm{~d}, J=8.5 \mathrm{~Hz}, 1 \mathrm{H}, \mathrm{H}_{6}\right), 6.93-6.92(\mathrm{~s}, J=1 \mathrm{~Hz}, 8$ $\left.\mathrm{Hz}, 1 \mathrm{H}, \mathrm{H}_{2^{\prime \prime}}\right), 6.90-6.88\left(\mathrm{~d}, J=1.5 \mathrm{~Hz}, 8 \mathrm{~Hz}, 1 \mathrm{H}, \mathrm{H}_{5^{\prime \prime}}\right)$, $6.84-6.82\left(\mathrm{~d}, J=8.5 \mathrm{~Hz}, 1 \mathrm{H}, \mathrm{H}_{6^{\prime}}\right), 6.56(\mathrm{~s}, J=1.5 \mathrm{~Hz}$, $\left.1 \mathrm{H}, \mathrm{H}_{3}\right), 6.46-6.44\left(\mathrm{~d}, J=2.5 \mathrm{~Hz}, 8 \mathrm{~Hz}, 1 \mathrm{H}, \mathrm{H}_{5}\right), 5.87$ (s, 1H, NH), $4.82-4.78\left(\mathrm{t}, 1 \mathrm{H}, \mathrm{H}_{3}\right), 3.87(\mathrm{~s}, 6 \mathrm{H}, 2 \times \mathrm{Ar}-$ $\left.\mathrm{OCH}_{3}\right), 3.81\left(\mathrm{~s}, 3 \mathrm{H}, \mathrm{Ar}-\mathrm{OCH}_{3}\right), 3.52-3.47\left(\mathrm{q}, 1 \mathrm{H}, \mathrm{H}_{4 \beta}\right)$, $3.09-3.05\left(\mathrm{q}, 1 \mathrm{H}, \mathrm{H}_{4 \alpha}\right)$.

\subsubsection{N,N-Dimethyl-4-(3-(4-nitrophenyl)-4,5- dihydro-1H-pyrazol-5-yl)-benzenamin (5b)}

4'-Nitro-4-dimethylaminochalcone (4b, 2 mmol) was dissolved in $100 \mathrm{ml}$ absolute ethanol. To this mixture, hydrazine hydrate $(4 \mathrm{mmol})$ was added dropwise at room temperature. The reaction mixture was refluxed for 7 hours, monitoring by TLC with solvent system of n-hexaneacetone (3:1). The reaction mixture was cooled in ice bath. The resulting white solid was filtered and washed with cold water. The filtrate was extracted with ether and evaporated in vacuo yielding more solid. The solid was collected and crystalized from ethanol to yield N,N-di-
methyl-4-(3-(4-nitrophenyl)-4,5-dihydro-1H-pyrazol-5yl)-benzenamin (5b). White solid, $\mathrm{mp}: 154^{\circ} \mathrm{C}-154.7^{\circ} \mathrm{C}$, IR $(\mathrm{KBr}), v\left(\mathrm{~cm}^{-1}\right): 3334(\mathrm{~N}-\mathrm{H}), 1614(\mathrm{C}=\mathrm{N}) .{ }^{1} \mathrm{H}-\mathrm{NMR}$ $\left(500 \mathrm{MHz}, \mathrm{CDCl}_{3}\right): \delta 8.22-8.20(\mathrm{~d}, J=2 \mathrm{~Hz}, 9 \mathrm{~Hz}, 2 \mathrm{H}$, $\left.\mathrm{H}_{3^{\prime}}, \mathrm{H}_{4^{\prime}}\right), 7.78-7.76\left(\mathrm{~d}, J=1.5 \mathrm{~Hz}, 9 \mathrm{~Hz}, 2 \mathrm{H}, \mathrm{H}_{2}, \mathrm{H}_{6^{\prime}}\right.$ ), $7.21-7.19$ (d, $\left.J=8.5 \mathrm{~Hz}, 2 \mathrm{H}, \mathrm{H}_{2}{ }^{\prime}, \mathrm{H}_{6}{ }^{\prime}\right), 6.71-6.69$ (d, $J=$ $\left.2 \mathrm{~Hz}, 9 \mathrm{~Hz}, 2 \mathrm{H}, \mathrm{H}_{3^{\prime}}, \mathrm{H}_{4^{\prime}}\right), 6.19$ (s, 1H, NH), 4.95 - 4.92 (t, $\left.1 \mathrm{H}, \mathrm{H}_{3}\right), 3.45-3.39$ (dd, $\left.1 \mathrm{H}, \mathrm{H}_{4}\right), 2.94\left(\mathrm{~s}, 6 \mathrm{H}, 2 \times \mathrm{CH}_{3}\right)$.

\subsubsection{2-(5-(2,4-Dimethoxyphenyl)-4,5-dihydro- 1H-pyrazol-3-yl)-phenol (5c)}

2'-Hydroxy-2,4-dimethoxychalcone (4c, $2 \mathrm{mmol}$ ) was dissolved in $100 \mathrm{ml}$ absolute ethanol. To this mixture, hydrazine hydrate $(4 \mathrm{mmol})$ was added dropwise at room temperature. The reaction mixture was refluxed for 7 hours, monitoring by TLC with solvent system of nhexane-acetone (3:1). The reaction mixture was cooled in ice bath. The resulting white solid was filtered and washed with cold water. The filtrate was extracted with ether and evaporated in vacuo yielding more solid. The solid was collected and crystalized from ethanol to yield 2-(5-(2,4-dimethoxyphenyl)-4,5-dihydro-1H-pyrazol-3-yl)phenol (5c). White solid, mp: $154^{\circ} \mathrm{C}-154.7^{\circ} \mathrm{C}$, IR (KBr), $v\left(\mathrm{~cm}^{-1}\right): 3327(\mathrm{~N}-\mathrm{H}), 1591(\mathrm{C}=\mathrm{N}), 1153(\mathrm{C}-\mathrm{O}) .{ }^{1} \mathrm{H}-\mathrm{NMR}$ $\left(500 \mathrm{MHz}, \mathrm{CDCl}_{3}\right): \delta 11.09$ (s, $\left.1 \mathrm{H}, \mathrm{Ar}-\mathrm{OH}\right), 7.24-7.18$ $\left(\mathrm{m}, 3 \mathrm{H}, \mathrm{H}_{3}, \mathrm{H}_{4}, \mathrm{H}_{6}\right), 6.99-6.97(\mathrm{~d}, J=1 \mathrm{~Hz}, 8 \mathrm{~Hz}, 1 \mathrm{H}$, $\left.\mathrm{H}_{6}{ }^{\prime}\right), 6.87-6.84$ (t, $\left.J=8.5 \mathrm{~Hz}, 1 \mathrm{H}, \mathrm{H}_{5^{\prime}}\right), 6.47(\mathrm{sb}, 1 \mathrm{H}, \mathrm{NH})$, 6.47 - $6.44\left(\mathrm{~m}, 2 \mathrm{H}, \mathrm{H}_{3^{\prime \prime}}, \mathrm{H}_{5^{\prime \prime}}\right), 5.13-5.09\left(\mathrm{t}, 1 \mathrm{H}, \mathrm{H}_{3}\right), 3.82$ (s, $\left.3 \mathrm{H}, \mathrm{Ar}-\mathrm{OCH}_{3}\right), 3.79$ (s, $\left.3 \mathrm{H}, \mathrm{Ar}-\mathrm{OCH}_{3}\right), 3.50-3.45$ (q, $\left.1 \mathrm{H}, \mathrm{H}_{4 \beta}\right), 3.08$ - 3.03 (q, 1H, $\mathrm{H}_{4 \alpha}$ ).

\subsubsection{2-Allyloxybenzaldehyde (8)}

To a solution of salicylaldehyde $(6,0.1 \mathrm{~mol})$ and allyl bromide $(7,0.11 \mathrm{~mol})$ in anhydrous acetone $(30 \mathrm{ml})$, potassium carbonate $(0.12 \mathrm{~mol})$ was added. The reaction mixture was refluxed for 3 hours. At the completion of the reaction (monitoring by TLC with solvent system of n-hexane-acetone 3:1), the resulting mixture was cooled to room temperature and poured into water $(150 \mathrm{ml})$. The aqueous phase was then extracted with dichloromethane. The obtained extract was washed with $5 \%$ sodium hydroxide $(\mathrm{NaOH})$ solution and water. The organic phase was dried over anhydrous magnesium sulfate and concentrated under reduced pressure. After distillation under reduced pressure, 2-allyloxybenzaldehyde (8) was obtained as a yellow liquid. Yield $87 \%$, boiling point (bp): $273.5^{\circ} \mathrm{C} / 760 \mathrm{mmHg} .{ }^{1} \mathrm{H}-\mathrm{NMR}\left(500 \mathrm{MHz}, \mathrm{CDCl}_{3}\right): \delta 4.75$ $(\mathrm{dd}, J=5.0 \mathrm{~Hz}, 1.6 \mathrm{~Hz}, 2 \mathrm{H}), 5.13-5.43(\mathrm{~m}, 2 \mathrm{H}), 5.4-$ $5.9(\mathrm{~m}, 1 \mathrm{H}), 6.65-8.12(\mathrm{~m}, 4 \mathrm{H}), 10.62(\mathrm{~s}, 1 \mathrm{H})$.

\subsubsection{2-Hydroxy-3-allylbenzaldehyde (9)}

$14 \mathrm{~g}$ of 2-allyloxybenzaldehyde (8) was heated in a sealed tube. After 12 hours, the rearrangement was complete. 
The reaction was cooled to room temperature and diluted with $50 \mathrm{ml}$ diethyl ether. The diluted solution was then extracted with $10 \% \mathrm{NaOH}$. The alkaline extract was added $10 \% \mathrm{HCl}$ until the solution became slightly acidic $(\mathrm{pH} \mathrm{6)}$. The above solution was then extracted with diethyl ether. The ethereal extract was dried with anhydrous magnesium sulfate and was concentrated in vacuo. The oily residue was distilled under reduced pressure to get 2-hydroxy-3-allylbenzaldehyde (9) as a light yellow liquid. Yield $72.8 \%(10.2 \mathrm{~g})$, bp: $243.5^{\circ} \mathrm{C} / 760 \mathrm{mmHg}$. ${ }^{1} \mathrm{H}-\mathrm{NMR}\left(500 \mathrm{MHz}, \mathrm{CDCl}_{3}\right): \delta 11.30(\mathrm{~s}, 1 \mathrm{H}, \mathrm{Ar}-\mathrm{OH})$, 9.85 (s, 1H, CHO), 7.41 - 7.39 (m, 2H, $\left.\mathrm{H}_{4}, \mathrm{H}_{6}\right), 6.97$ $6.94\left(\mathrm{t}, 1 \mathrm{H}, \mathrm{H}_{5}\right), 6.03-5.95\left(\mathrm{~m}, 1 \mathrm{H}, \mathrm{H}_{2}\right), 5.12-5.08(\mathrm{~m}$, $\left.2 \mathrm{H},=\mathrm{CH}_{2}\right), 3.44-3.41\left(\mathrm{~d}, 1 \mathrm{H}, \mathrm{H}_{1}{ }^{\circ}\right)$. Compound 9 was used as the material for the synthesis of compounds $11 \mathrm{a}-\mathrm{b}$.

\subsubsection{2'-Hydroxy-5'-methyl-2-hydroxy-3- allylchalcone (11a)}

2-Hydroxy-5-methylacetophenone (1.05 equivalence) and 2-hydroxy-3-allylbenzaldehyde (9) were dissolved in methanol. To the above mixture, potassium hydroxide (3 equivalence) was added in portions to give a blood-red solution. The reaction mixture was stirred at room temperature for 52 hours, during which 2'-hydroxy-5'-methyl-2-hydroxy-3-allylchalcone (11a) precipitated as the potassium salt. The reaction mixture was poured into cold $1 \mathrm{~N}-\mathrm{HCl}$ solution and was further added c- $\mathrm{HCl}$ until the solution became acidic. The resulting precipitate was filtered, washed with water, and crystalized from methanol to give product $11 \mathrm{a}$ as crystals. Yield $40 \%$. Light purple solid, $\mathrm{mp}: 156^{\circ} \mathrm{C}, \mathrm{IR}(\mathrm{KBr}), v\left(\mathrm{~cm}^{-1}\right): 1706(\mathrm{C}=\mathrm{O})$, $1559(\mathrm{C}=\mathrm{C}$ aryl $), 1166(\mathrm{C}-\mathrm{O}) .{ }^{1} \mathrm{H}-\mathrm{NMR}(500 \mathrm{MHz}$, $\mathrm{CDCl}_{3}$ ): $\delta 12.65$ (s, $\left.1 \mathrm{H}, \mathrm{Ar}-\mathrm{OH}\right), 8.20-8.17$ (d, $J=15.5$ $\left.\mathrm{Hz}, 1 \mathrm{H}, \mathrm{H}_{\beta}\right), 7.71-7.68\left(\mathrm{~d}, J=15.5,1 \mathrm{H}, \mathrm{H}_{\alpha}\right), 7.66(\mathrm{~s}, 1 \mathrm{H}$, $\left.\mathrm{H}_{6}\right), 7.51-7.49\left(\mathrm{~d}, J=1 \mathrm{~Hz}, 7.5 \mathrm{~Hz}, 1 \mathrm{H}, \mathrm{H}_{6}\right), 7.28(\mathrm{~s}, J$ $\left.=2 \mathrm{~Hz}, 1 \mathrm{H}, \mathrm{H}_{2}\right), 7.16-7.14(\mathrm{~d}, J=1 \mathrm{~Hz}, 7.5 \mathrm{~Hz}, 1 \mathrm{H}$, $\left.\mathrm{H}_{4}\right), 7.04-7.02\left(\mathrm{~d}, J=1 \mathrm{~Hz}, 8 \mathrm{~Hz}, 1 \mathrm{H}, \mathrm{H}_{3}\right), 6.91-6.88$ $\left(\mathrm{t}, 1 \mathrm{H}, \mathrm{H}_{5}\right), 6.89-6.87\left(\mathrm{~d}, J=8.5 \mathrm{~Hz}, 1 \mathrm{H}, \mathrm{H}_{4}\right), 6.03-$ $5.95\left(\mathrm{~m}, 1 \mathrm{H}, \mathrm{H}_{2}{ }^{\prime \prime}\right), 5.16-5.12\left(\mathrm{~m}, 2 \mathrm{H},=\mathrm{CH}_{2}\right), 3.42-3.41$ (d, $1 \mathrm{H}, \mathrm{H}_{1}$ ), 2.30 (s, 3H, $\mathrm{Ar}-\mathrm{CH}_{3}$ ).

\subsubsection{4'-Nitro-2-hydroxy-3-allylchalcone (11b)}

4'-Nitroacetophenone (1.05 equivalence) and 2-hydroxy3 -allylbenzaldehyde (9) were dissolved in methanol. To the above mixture, potassium hydroxide (3 equivalence) was added in portions to give a blood-red solution. The reaction mixture was stirred at room temperature for 50 hours, during which 4'-nitro-2-hydroxy-3-allylchalcone (11b) precipitated as the potassium salt. The reaction mixture was poured into cold $1 \mathrm{~N}-\mathrm{HCl}$ solution and was further added $\mathrm{c}-\mathrm{HCl}$ until the solution became acidic. The resulting precipitate was filtered, washed with water, and crystalized from methanol to give product $11 \mathrm{~b}$ as crystals. Yield $45 \%$. Light purple solid, mp: $180^{\circ} \mathrm{C}$, IR $(\mathrm{KBr}), v$ $\left(\mathrm{cm}^{-1}\right)$ : $1651(\mathrm{C}=\mathrm{O}), 1573(\mathrm{C}=\mathrm{C}$ aryl $), 1170(\mathrm{C}-\mathrm{O}) .{ }^{1} \mathrm{H}-$ NMR $\left(500 \mathrm{MHz}, \mathrm{CDCl}_{3}\right): \delta 8.28-8.26(\mathrm{~d}, J=8.5 \mathrm{~Hz}$, $\left.2 \mathrm{H}, \mathrm{H}_{3}, \mathrm{H}_{4}\right), 8.141-8.11\left(\mathrm{~d}, J=16 \mathrm{~Hz}, 1 \mathrm{H}, \mathrm{H}_{\beta}\right), 8.08-$ 8.06 (d, $\left.J=8.5 \mathrm{~Hz}, 2 \mathrm{H}, \mathrm{H}_{2}, \mathrm{H}_{6}\right), 7.53-7.49$ (d, $J=16 \mathrm{~Hz}$, $\left.1 \mathrm{H}, \mathrm{H}_{\alpha}\right), 7.47-7.45\left(\mathrm{~s}, J=8 \mathrm{~Hz}, 1 \mathrm{H}, \mathrm{H}_{6}\right), 7.15-7.13$ (d, $\left.J=7.5 \mathrm{~Hz}, 1 \mathrm{H}, \mathrm{H}_{4}\right), 6.88-6.86\left(\mathrm{t}, 1 \mathrm{H}, \mathrm{H}_{5}\right), 5.98-5.93$ $\left(\mathrm{m}, 1 \mathrm{H}, \mathrm{H}_{2}{ }^{\prime}\right), 5.12-5.07\left(\mathrm{~m}, 2 \mathrm{H},=\mathrm{CH}_{2}\right), 3.39-3.38(\mathrm{~d}$, $\left.1 \mathrm{H}, \mathrm{H}_{1}{ }^{\prime \prime}\right)$.

\subsection{Biological Evaluation}

\subsubsection{Antimicrobial Testing}

Compounds 4/5a-c and 11a-b were evaluated for their in vitro antimicrobial activity by agar disc-diffusion method [16]. Stock solutions of test compounds were diluted in dimethyl sulfoxide (DMSO) (1\%) to give a final concentration of $1024 \mu \mathrm{g} / \mathrm{ml}$. The DMSO (1\%) alone was used as a control. Sterile filter paper discs (6 $\mathrm{mm}$ diameter) moistened with the test compound solution were carefully placed on the agar culture plates which had been previously inoculated separately with the microorganisms. The plates were incubated at $37^{\circ} \mathrm{C}$ for 24 hours (in the case of bacteria) or 48 hours (in the case of fungi). After incubation, growth was surveyed by measuring the diameter of the growth inhibition zones. All determinations were made in triplicate for each compound. Average of three independent readings for each compound was recorded. The results are given in Table 1.

MIC was defined as the lowest concentration of compound required for a complete inhibition of inoculated bacteria or fungi after incubation time. Sulfamethoxazole and Ketoconazole were used as reference agents for antibacterial and antifungal activities respectively. The MICs of the test compounds were determined using microdilution method [17]. The test compounds, Sulfamethoxazole and Ketoconazole were dissolved in DMSO (1\%) to give a concentration of $1024 \mu \mathrm{g} / \mathrm{ml}$; and two-fold dilution of the solution was prepared $(512,256,128,64,32$, $16,8$, and $4 \mu \mathrm{g} / \mathrm{ml})$. The microorganism suspensions were inoculated to the corresponding wells. The plates were incubated at $37^{\circ} \mathrm{C}$ for 24 hours (in the case of bacteria) or 48 hours (in the case of fungi). The MIC values are displayed in Table 2.

\subsubsection{Antioxidant Testing}

Compounds 4/5a-c and 11a-b were tested for antioxidant activity by DPPH radical scavenging method [18]. The nitrogen centered stable free radical DPPH has been in widespread use in spectrophotometric studies to characterize antioxidants [19]. This is based on the fact that the odd electron in the DPPH free radical gives a strong ab- 
sorption maximum at $\lambda 517 \mathrm{~nm}$, which is purple in color [18]. A radical scavenging antioxidant reacts with DPPH stable free radical, resulting in the decolorization which is stoichiometric with respect to the number of electrons captured [18]. The change on the absorbance produced in this reaction is used to measure antioxidant properties [18].

Stock solutions of different compounds $(1 \mathrm{mM})$ were mixed with $0.5 \mathrm{ml}$ of $0.3 \mathrm{mMDPPH}$ in methanol. Final volume was adjusted to $3 \mathrm{ml}$. Reaction mixtures were variously shaken and allowed to react for 30 minutes in the dark at room temperature. Absorbance values were measured at $517 \mathrm{~nm}$ using anultraviolet-visible (UV-VIS) spectrophotometer. $0.5 \mathrm{ml}$ of $0.3 \mathrm{mM}$ DPPH diluted in $2.5 \mathrm{ml}$ of methanol was used as control. Absorbance was converted to $\%$ antioxidant activity using the following equation: $\mathrm{S}(\%)=100\left(\mathrm{~A}_{0}-\mathrm{A}_{\mathrm{S}}\right) / \mathrm{A}_{0}$ where $\mathrm{A}_{0}$ is the absorbance of the control (containing all reagents except the test compound) and $A_{S}$ represents absorbance of the test compound. Results were compared with activity of vitamin $\mathrm{C}$ which was used as the standard. The data are summarized in Table 3.

\section{Authors' Disclosures of Potential Conflicts of Interest}

The authors indicated no potential conflicts of interest.

\section{REFERENCES}

[1] E. Grotewold, "The Science of Flavonoids," Springer, New York, 2006. doi:10.1007/978-0-387-28822-2

[2] Y. M. Lin, Y. Zhou, M. Flavin, L. M. Zhou, W. Nie and F. C. Chen, "Chalcones and Flavonoids as Anti-Tuberculosis Agents," Bioorganic \& Medicinal Chemistry, Vol. 10, No. 8, 2002, pp. 2795-2802. doi:10.1016/S0968-0896(02)00094-9

[3] M. L. Go, X. Wu and X. L. Liu, "Chalcones: An Update on Cytotoxic and Chemoprotective Properties," Current Medicinal Chemistry, Vol. 12, No. 4, 2005, pp. 483-499.

[4] Z. Nowakowska, "A Review of Anti-Infective and AntiInflammatory Chalcones," European Journal of Medicinal Chemistry, Vol. 42, No. 2, 2007, pp. 125-137. doi:10.1016/i.ejmech.2006.09.019

[5] S. Velavan, K. Nagulendran, R. Mahesh and V. Begum, "In Vitro Antioxidant Activity of Asparagus racemosusroot," Pharmacognosy Magazine, Vol. 3, No. 9, 2007, pp. 26-33.

[6] M. Okawa, J. Kinjo, T. Nohara and M. Ono, "DPPH (1,1-Diphenyl-2-picrylhydrazyl) Radical Scavenging Activity of Flavonoids Obtained from Some Medicinal Plants," Biological and Pharmaceutical Bulletin, Vol. 24, No. 10, 2001, pp. 1202-1205. doi:10.1248/bpb.24.1202

[7] E. Cadenas and L. Packer, "Handbook of Antioxidants," Marcel Dekker, New York, 1996.
[8] C. Rice-Evans and L. Packer, "Flavonoids in Health and Disease," Marcel Dekker, New York, 2003.

[9] O. Prakash, R. Kumar and V. Parkash, "Synthesis and Antifungal Activity of Some New 3-Hydroxy-2-(1-phenyl3-aryl-4-pyrazolyl)chromones", European Journal of Medicinal Chemistry, Vol. 43, No. 2, 2008, pp. 435-440. doi:10.1016/j.ejmech.2007.04.004

[10] O. Prakash, R. Kumar and R. Sehrawat, "Synthesis and Antibacterial Activity of Some New 2,3-Dimethoxy-3-hydroxy-2-(1-phenyl-3-aryl-4-pyrazolyl)chromanones," European Journal of Medicinal Chemistry, Vol. 44, No. 4, 2009, pp. 1763-1767. doi:10.1016/j.ejmech.2008.03.028

[11] B. Bandgar, S. Gawande, R. Bodade, N. Gawande and C. Khobragade, "Synthesis and Biological Evaluation of a Novel Series of Pyrazolechalcones as Anti-Inflammatory, Antioxidant and Antimicrobial Agents," Bioorganic \& Medicinal Chemistry, Vol. 17, No. 24, 2009, pp. 81688173. doi:10.1016/i.bmc.2009.10.035

[12] H. Adibi, J. S. Mojarrad, H. Asgharloo and G. Zarrini, "Synthesis, in Vitro Antimicrobial and Antioxidant Activities of Chalcone and Flavone Derivatives Holding Allylic Substitutions," Medicinal Chemistry Research, Vol. 20, No. 8, 2010, pp. 1318-1324.

[13] N. S. Joshi, A. A. Shaikh, A. P. Deshpande, B. K. Karale, S. B. Bhirud and C. H. Gill, "Synthesis, Characterization and Antimicrobial Activities of Some Fluorine Containing 2-(1-Phenyl-3-aryl-1H-pyrazol-4-yl)-3-chlorochromones, 2-(1-Phenyl-3-aryl-1H-pyrazol-4-yl)chromones and 5-(1-Phenyl-3-aryl-1H-pyrazol-4-yl)-3-(2-hydroxyphenyl)4,5-dihydropyrazolines," Indian Journal of Chemistry, Vol. 44B, No. 2, 2005, pp. 422-425.

[14] T. T. Dao, Y. S. Chi, J. Kim, H. P. Kim, S. Kim and H. Park, "Synthesis and PGE2 Inhibitor Activity of 5,7-Dihydroxyflavones and Their $O$-Methylated Flavone Analogs," Archives of Pharmacal Research, Vol. 26, No. 5, 2003, pp. 345-350. doi:10.1007/BF02976690

[15] M. Hiersemann and U. Nubbemeyer, "The Claisen Rearrangement: Methods and Applications," Wiley-VCH Verlag $\mathrm{GmbH}$, Weinheim, 2007.

[16] National Committee for Clinical Laboratory Standards (NCCLS), "Approved Standard Document M-7A," Villanova, P.A., 1985.

[17] P. R. Murray, E. Baron, J. Jorgensen, M. Landry and M. Pfaller, "Manual of Clinical Microbiology," 9th Edition, American Society of Microbiology, Washington DC, 2007.

[18] K. Kato, S. Terao, N. Shimamoto and M. Hirata, "Studies on Scavengers of Active Oxygen Species. 1. Synthesis and Biological Activity of 2-O-Alkylascorbic Acids," Journal of Medicinal Chemistry, Vol. 31, No. 4, 1988, pp. 793-798. doi:10.1021/jm00399a019

[19] Padmaja, T. Payani, G. D. Reddy and V. Padmavathi, "Synthesis, Antimicrobial and Antioxidant Activities of Substituted Pyrazoles, Isoxazoles, Pyrimidine and Thioxopyrimidine Derivatives," European Journal of Medicinal Chemistry, Vol. 44, No. 11, 2009, pp. 4557-4566. doi:10.1016/i.ejmech.2009.06.024 